\title{
Potential harms, anonymization, and the right to withdraw consent to biobank research
}

\author{
Stefan Eriksson*,1 and Gert Helgesson ${ }^{1}$ \\ ${ }^{1}$ Centre for Bioethics at Karolinska Institutet and Uppsala University, Uppsala Science Park, SE-751 85 Uppsala, \\ Sweden
}

This paper discusses the potential harms involved in biobank research and how ethical review, informed consent, withdrawals, and anonymization of samples should be handled in the light of these harms. There is less risk involved in biobank research than in human subject research; it should therefore be treated differently. In our view, anonymization should not be an automatically permissible response to requests for withdrawal. Nor should a request for withdrawal necessarily stop research on identifiable samples. Apart from not being particularly appropriate for protecting the interests of individuals, anonymization of samples has a negative impact on research. We suggest that the current view on withdrawal from research, supported by the Declaration of Helsinki and subsequent ethical guidelines, be abandoned in the context of biobank research and be replaced by an approach inspired by the Nuremberg Code. This approach requires those wishing to withdraw their samples from research to present sufficient reason for doing so. Our interpretation of 'sufficient reason' includes all those involving genuine, deeply felt concerns that are not based on misconceptions. Still, this underlines the fact that we all share a responsibility for health research and that no one should take withdrawal from biobank research lightly.

European Journal of Human Genetics (2005) 13, 1071-1076. doi:10.1038/sj.ejhg.5201458;

published online 29 June 2005

Keywords: anonymization; biobank research; harm; informed consent; Nuremberg Code; withdrawal of consent

\section{Introduction}

Risks involved in biobank $^{1}$ research have been widely discussed lately. New ethical guidelines have been introduced in order to protect sample donors from harm. ${ }^{2-5}$ New legal regulations have been enacted in, for example, Iceland, Norway, Sweden, and the UK. Other European countries, for instance Finland, have adjusted previous legislation to also cover research on archived biological material. However, further analysis is needed to clarify what negative effects biobank research may have and what recommendations regarding ethical review, informed con-

${ }^{*}$ Correspondence: $\operatorname{Dr} S$ Eriksson, Centre for Bioethics at Karolinska Institutet and Uppsala University, Uppsala Science Park, SE-751 85 Uppsala, Sweden. Tel: + 461861122 87; Fax: + 46185064 04; E-mail: Stefan.Eriksson@bioethics.uu.se

Received 4 March 2005; revised 18 May 2005; accepted 24 May 2005; published online 29 June 2005 sent, and procedures for the storage and use of biobank samples are needed in order to counter or reduce these risks. Part of such an analysis will be undertaken in this paper.

A presumption in much of the debate, reflected in legal regulations and ethical guidelines, is that potential problems connected with biobank research are related to the harm that may arise if sensitive personal information ends up in the wrong hands. When participants wish to withdraw from a study, de-identification of samples and data (making them unlinkable to specific individuals) has therefore been considered a satisfactory solution. On the contrary, we argue that anonymization should not be an automatically permissible response to requests for withdrawal; nor should a request for withdrawal necessarily stop research on identifiable samples. Apart from not being particularly appropriate for protecting the interests of 
individuals, anonymization of samples has a negative impact on research. We suggest that the current view on withdrawal from research be abandoned in the context of biobank research and be replaced by an approach inspired by the Nuremberg Code.

\section{Risks of harm related to biobank research}

There is a certain risk of physical harm connected to the taking of samples, the amplitude depending on the kind of sample and physical state of the sample donor. Storing a sample in a biobank or using it for research, however, involves no risk of direct physical harm. As recognized by the German Nationaler Ethikrat, bodily risks are precluded from the outset because the samples used for research have already been separated from the body. ${ }^{4}$ In this respect, there is a difference between biobank research and research conducted directly on humans. This has often been overlooked in policy work. Thus, the Declaration of Helsinki states that medical research involving human subjects 'includes research on identifiable human material or identifiable data' and makes no distinction between research of the latter kind and experimental research when it comes to requirements regarding information and consent. For instance, according to the declaration, every research subject has the right to withdraw consent to participate at any time without reprisal, regardless of whether the research is conducted on their bodies or on their stored biological samples. ${ }^{5}$

Although there is no risk of direct physical harm involved in biobank research, there is a palpable risk of indirect physical harm. Most samples are originally collected in a clinical setting and may turn out to be important for the future care of the patients. If researchers are granted unrestricted access, samples may be exhausted completely or become too sparse for future purposes of care, having possible negative effects on some participants' physical health.

Biobank research may also involve a risk of various forms of nonphysical harm. Nonphysical harm is usually tied to sensitive information ending up in the wrong hands and being used to the disadvantage of a person. This information may be either extracted from the samples or tied to them. As long as safety routines for the biobank and the data collected therein are satisfactory, informational risk does exist but is small. The inflicted harm may be social, psychological, or economical.

For instance, it has been argued that insurance companies and employers could come to use genetic information to discriminate against people with certain genetic dispositions. This would likely damage the individuals concerned, both economically and socially. ${ }^{6}$ When facts one would like to keep secret (eg regarding paternity or STDs) are communicated to relatives or partners against one's wishes, psychological harm may arise. ${ }^{7}$ Psychological harm can also occur, for instance, when patients wrongly form the belief that there will be personal gain from participation in research (the 'therapeutic misconception $^{8}$ ). When such expectations are not met, feelings of anger or hopelessness can result. Much other potential nonphysical harm is easily imagined in the form of, for instance, stigmatization and discrimination at work as well as anxiety and disturbed relationships with spouses, children, relatives, and friends.

Not all risks of harm are related to specific individuals. ${ }^{9}$ Samples used in biobank research may harm the group to which a participant belongs, and thereby harm the individuals concerned. This harm may arise, for instance, if people outside the group regard the group in a more negative way or treat people belonging to that group in a worse way than others, or by prejudice being spread or strengthened. The harm may also be due to people in the group starting to look at themselves in a different, more negative, way.

Thus, a distinction can be made between external and internal harm to groups of people. External harm is connected to factors external to the group, such as changes in attitudes regarding the group. Such changes can cause economic harm, for instance more expensive insurance for group members due to expectations about their behaviour. Internal harm, on the other hand, is related to internal changes resulting from the spread of information from biobank research. Loss of self-esteem is an example of internal harm, decline in social functions and bonds another.

\section{Moral harms}

The types of harms discussed so far do not exhaust the possible negative effects of biobank research. Apart from harming participants, as mentioned above, one may wrong them by storing or using their samples. In other words, one may treat an individual in an illegitimate way even if they are not exposed to any risk of physical, psychological, social or economic harm. We call this 'moral harm'. It should be clear that the category of wrongs is relevant to the ethics of biobank research.

What kinds of wrongs are there in relation to the storage and use of biobank samples? In connection to medical research, as well as the procedures of informed consent usually treated as absolutely essential to ethically acceptable research, autonomy, privacy, and personal integrity are central values at stake. The term autonomy has been used in different ways. We take it to mean self-governance (selfdetermination); that is, deciding for oneself and acting on these decisions regarding matters affecting one's own life, without interference from others. Respecting someone's autonomy is respecting that person's right to decide and 
act in this way. The right to privacy is the right to a personal sphere, free from public attention and interference. It includes the right to keep certain facts about oneself secret. Personal integrity presupposes a certain degree of privacy as well as a certain degree of autonomy. Individuals' personal integrity is disrespected if others intrude into their private life or prevent them from making autonomous decisions about their life.

For instance, biobank research creates moral harm when samples are used for research that the participants would object strongly to, or when researchers by means of their access to samples and personal information draw conclusions regarding paternity, look into details of eating and drinking habits, or deal with other private matters that individuals wish to keep to themselves. If certain tissue holds special meaning and value to people (blood, placenta, or foetal tissue, for example), then they are wronged if that tissue is treated inconsiderately.

It is sometimes argued that you commit an additional wrong by violating someone's dignity. For example, deceased persons possess dignity (or a right to be treated with dignity) even in death, which should prevent doctors from performing certain procedures on a corpse. Accordingly, many guidelines and laws have special provisions regarding post mortem use of samples. Consistency demands that if dignity is relevant in our treatment of the dead, then it is relevant in our treatment of the living as well.

It should be noted that groups can also be wronged, even if no single individual is pointed out specifically. For instance, groups are treated badly when they are used for research because of their weak position or inability to protest. Groups are also wronged when treated unjustly, if they are systematically excluded from the benefits of research.

\section{Conflated models of research ethics}

It is important that there be a safeguard against the harms discussed above. The requirement of approval from a research ethics committee is therefore appropriate. However, that ethical reviews should be carried out does not mean that biobank research should have to face the same demands as human subject research does. On the contrary, it is quite reasonable to have different requirements since the potential harms differ.

We need to refrain from automatically subsuming biobank research and other kinds of database and register research under the 'experimentation' moniker. Otherwise, we risk conflating different models of research ethics. On the one hand, there is the Helsinki Declaration model on the integrity of the experimental subject. On the other hand, there are the ethical considerations made for epidemiology and register research. The absolute right to decide for oneself is virtually indispensable in the former case but seems rather out of place in the latter, for which no risk of direct physical harm exists. This differentiation is also acknowledged in some legal regulations regarding registers, in which the collection of personal information is mandatory by law. The individual person's interest in selfdetermination is then considered to be outweighed by public health interests in allowing for research to be carried out.

Actually, individuals can assert their interests not only by directly exercising their autonomy in various ways, but also by obtaining provision for their interests indirectly, through elected or appointed representatives. Thus, a distinction must be made between a direct and an indirect form of exercising individual rights and protecting individual interests. Empirical data show that participants in biobank research can be willing to hand over their power of decision-making to educated and experienced peers. ${ }^{10}$ Even when they are not willing to do so, it can still be the case that the interest of the individuals concerned in exercising their autonomy regarding participation is less important than research being carried out unobstructed.

One part of the human subjects research ethics that has been carried over to biobank research ethics is the farreaching right to withdraw one's consent. Instead of challenging this move, policymakers have suggested anonymization as a solution to the problems this 'right' creates.

\section{Anonymization as a response to request for withdrawal}

According to many regulations and guidelines, there is no need to return or destroy a sample when a participant withdraws from a study, since anonymization addresses the substantial ethical problem. The European Society of Human Genetics writes that the use of 'unlinked anonymized samples' secures 'absolute confidentiality' and these samples can thus be used for new purposes without the need to obtain consent. ${ }^{2}$ The International Bioethics Committee (UNESCO) states that consent to research may be withdrawn by the donor 'unless such data are irretrievably unlinked to an identifiable person' and that the data and biological samples should be dealt with in accordance with the wishes of the donor unless they are irretrievably unlinked. ${ }^{3}$ The German Nationaler Ethikrat maintains that if samples are anonymized, then 'donor interests calling for protection are not at issue'; therefore, withdrawal of consent need only be responded to with anonymization. ${ }^{4}$

The idea behind this solution is simply that if harms arise due to the spreading of information about specific individuals, then the risks of harm are eliminated altogether by irreversibly destroying the tie between information and individual. Information derived from your tissue or blood samples cannot be used to your disadvantage if no one knows, or can find out, that the information concerns 
you. You have the right to end your participation, but since it can be of importance to keep your sample for research, de-identification is performed. It is no longer 'you' who participates, and research can still be performed - all is well.

\section{Limited value of anonymization}

However, anonymization has a limited value in protecting participants' interests:

- It may not decisively cut the link to a specific individual,

- it prevents the use of samples for purposes such as diagnostics,

- it may not prevent harms to groups, and

- it does not rule out wrongdoing.

First, anonymizing samples in this context means making them irretrievably unlinked to sample donors by having all identifications removed. This can be done by destroying the code keys. We now know this is not enough to guarantee anonymity. If demographic and clinical data accompany the anonymized sample (as is explicitly assumed in the recommendations from the ESHG), it may still be possible in some cases for individuals possessing sufficient knowledge to identify a donor.

Further, as soon as genetic information is stored in medical journals, a supposedly anonymous sample can be run against a search of these journals, which may reveal whose sample it is. The publication of databases on the Internet, free for researchers to use, also presents dangers in this respect. If you have access to an identified sample, you can find more samples from the same person by searching databases consisting of anonymous samples and comparing them with yours. This is no far-fetched fantasy but rather something easily performed with the aid of available technology. Therefore, anonymization is not enough but must be accompanied by further measures to be fully effective, such as requiring previous authorization for all searches for matching samples or limiting access to samples.

Second, note that if a sample is de-identified, it can no longer be used for diagnostic purposes. Individuals have an interest in being protected from the dispersal of sensitive information regarding them, but they also have an interest in having their samples available to medical services when needed. Anonymization prevents this. If proper safeguards are in place, the availability interest should carry greater weight than the interest in having additional protection by anonymization.

Third, even if no single individual is indicated in research material, there is still a risk that research on an identified group may lead to undesirable consequences for group members. Researchers need to consider this risk before publishing their results. Much has been done regarding these matters in the work on ethical guidelines for research on indigenous populations and the like.

Fourth, imagine that all these obstacles have been taken care of. Even then, the issue of wrongdoing remains. Consider autonomy: Assume that you have two test tubes of blood stored in a biobank containing 30000 other blood samples. Also assume that, as a response to your request that your samples be destroyed, those responsible for the biobank instead remove your name from all labels and lists within the premises, as well as any other material used to tie your samples to your name. In what way can their reaction be said to be a proper response to your request? It certainly seems that anonymization does not address the problem in cases like this, as it does in relation to other kinds of harm. The matter no longer concerns risks tied to the use of your samples - risks that may be more or less eliminated - but the very use itself. If someone says 'Do not use my samples', that person's request has simply not been respected by the use of anonymization.

Apart from not being particularly appropriate for protecting the interests of participants, anonymization of biobank samples has a negative impact on research.

For many types of studies, such as longitudinal and epidemiological research, it is absolutely essential that researchers, or at least those in charge of the biobank, have access to the identity of the samples so they can be linked to medical journals and register data as well as data from previous analyses. For quality reasons, researchers may also need to return to the original repository.

Thus, the 'anonymization tool' does not do much moral work. A model in which continued use of identified samples is impossible if withdrawal is requested, and where a request to have one's sample destroyed results in something else (namely anonymization) is hardly satisfactory. On the one hand, donors' arbitrary requests to have their samples destroyed are allowed to hinder important research. On the other hand, no matter what reasons people may have in requesting the destruction of a sample or how strong these reasons may be, their request can be met with a denial and continued research on that sample. Thus, anonymization involves a double moral limitation.

We again suggest that we meet these difficulties because we use the model of research on living human subjects for research on human body parts, tissue, and DNA. Is there an alternative?

\section{Restricted right to withdraw consent}

The demand for unrestricted individual rights to withdraw consent to participation in research is strongly connected to the Declaration of Helsinki from 1964. The notion of an individual's right to stop participation was already very important in the Nuremberg Code of 1947, but there it was conceived rather differently. While the Helsinki Declaration and subsequent documents have given the individual 
the right to withdraw regardless of reason, the Nuremberg Code conceived of research as a common good and saw experimental subjects as participants in an important humanitarian project, which led to a different view of the right to withdraw. Clause nine of the code states:

During the course of the experiment the human subject should be at liberty to bring the experiment to an end if he has reached the physical or mental state where continuation of the experiment seems to him to be impossible. ${ }^{11}$

There are good reasons why this notion of consent and withdrawal gave way to the view expressed later in the Helsinki Declaration, with its greater emphasis on voluntarism and freedom of choice. However, the Nuremberg view is better suited to biobank research than to research on human subjects and, we argue, preferable to the Helsinki view regarding biobank research.

Biobank research shares an important characteristic with register research and the like, namely that of being a public endeavour to promote the common good. This lends weight to the notion that there is a moral obligation to accept that one's biological samples are stored for and used in future biobank-related medical research. When you use modern medical services, you take advantage of the work done by prior generations. Why should you gain from this and not give something back to future patients? If you expect to receive the best possible treatment, you ought to contribute to the processes by which such treatment is established. If you do not, you are a free rider. ${ }^{12,13}$ This line of reasoning can be found among research participants. In Swedish biobank research, many patients express their willingness to contribute to research because they sense a duty to do so. ${ }^{14,15}$

A perceived duty to contribute may result in mandatory inclusion of personal data or biological samples in registers and collections. Our deduction is more modest: Those who have consented to the use of their biological samples in research should not be allowed to hinder that research later without providing valid reasons as to why they have changed their mind. If you have consented to a project that promotes the public good and if resources would be wasted if the research were not completed, you should at least be able to argue your case before the request to withdraw is granted. This gives the view of consent and withdrawal a contractual touch: To break a contract you usually need to point out the way in which the relationship between parties is different from what was assumed or expected when the contract was originally signed.

\section{Our proposal}

We propose that, instead of the present emphasis on individuals' right to withdraw consent to research on their biological samples for any reason, the clause on withdrawal in the Nuremberg Code be included in guidelines on biobank research. This would allow the available responses to a request for having one's samples withdrawn from research to be (1) anonymization, (2) further research on identifiable material and/or data, and (3) return to an original repository or destruction of the sample(s). To reflect the different context, the previously cited Nuremberg clause could be rewritten as follows:

During the course of the research, a participant should be at liberty to withdraw his consent if he can present sufficient reasons why it is no longer reasonable to ask for his continued participation. When such reasons are presented his sample(s) should be returned, destroyed or de-identified, according to the wish of the participant. If sufficient reasons are lacking, continued research is permissible.

Whether or not the reasons to withdraw are sufficient should be judged primarily by the researchers or biobank holders. However, if they are inclined to believe that the reasons are insufficient, then consultation with a research ethics committee is recommended. Certain variations between countries due to cultural differences can be expected in these kinds of decisions. However, we feel that genuine, deeply felt concerns (ie those in line with the person's character), not based on misconceptions, should generally be accepted as sufficient reason. If the concerns are based on misconceptions, then these misconceptions should be removed, if possible, by informing and explaining to those concerned.

This suggested approach can be motivated in terms of both personal integrity and potential psychological harm, since it is more likely that the wishes of the withdrawing individual will be listened to (because requests of withdrawal will be responded to less often by anonymization of the samples). It also better protects research interests since de-identification of samples is likely to occur less often (because requests of withdrawal will not automatically be accepted).

This approach can be supported from the perspective of trustworthiness and trust as well. If it is well established and made public that those who strongly object to participation in research for any genuinely felt reason (not based on misconception) will be allowed to withdraw and will then have the right to decide what will happen to their samples, then this is likely to have a positive effect on trust in research. Trustworthiness will also be promoted by the use of routines that do not take lightly the public health interests in research.

We also feel that any measure that allows people to be more involved in important research, so that they not only permit themselves to be subjected to it but encompass it as an important goal for themselves and for others, will 
benefit them as well as society at large. It will benefit the individual by fostering authenticity, and it will benefit society by creating a positive environment for research and development.

\section{Conclusions}

In this paper we have discussed the potential harms involved in biobank research and how informed consent, withdrawals, and anonymization of samples should be handled in the light of these potential harms. The kinds of harms relevant in this context are indirect physical harm and a number of nonphysical harms: economic, psychological, social, and moral. Noteworthy is that a person may be harmed by biobank research even if unexposed to economic, psychological, social, or indirect physical harm, namely if they are wronged.

Since biobank research does not involve any risk of direct physical harm, and is likely to involve nonphysical harms of different magnitude as compared to research on living human subjects, it is reasonable to treat it as a special kind of research more akin to register research. Since it may be reasonable to treat different kinds of research differently, it is by no means clear that guidelines relevant to research on human subjects are also relevant to biobank research.

We argue that anonymization should not be an automatically permissible response from researchers and biobank holders to requests of withdrawal. Nor should a request for withdrawal necessarily stop research on identifiable samples. Apart from not being particularly appropriate for protecting the interests of individuals, anonymization of biobank samples has a negative impact on important research.

We further suggest that the view on withdrawal from research supported by the Declaration of Helsinki and subsequent ethical guidelines be abandoned in the context of biobank research and be replaced by an approach more closely related to the Nuremberg Code. According to this approach, those who wish to withdraw their samples from research must present a sufficient argument for doing so, out of consideration of fairness and a duty to contribute to the continuous development of public health resources. Our interpretation of sufficient reason, however, is generous and includes all those with genuine, deeply felt concerns that are not based on misconceptions. Still, it underlines the importance of the issue and the fact that health research is a responsibility shared by researchers and the public. Donors should not take withdrawals from biobank research lightly.

\section{Acknowledgements}

Financial support from Wallenberg Consortium North is gratefully acknowledged.

\section{References}

1 Cambon-Thomsen A: The social and ethical issues of postgenomic human biobanks. Nat Rev Gen 2004; 5: 866-873.

2 Godard B, Schmidtke J, Cassiman JJ, Ayme S: Data storage and DNA banking for biomedical research: informed consent, confidentiality, quality issues, ownership, return of benefits. A professional perspective. Eur J Hum Genet 2003; 11 (Suppl 2): S88-S122.

3 UNESCO: International declaration on human genetic data. Eur J Health Law 2004; 11: 93-107.

4 Nationaler Ethikrat: Biobanks for research, opinion. Berlin: Nationaler Ethikrat, 2004.

5 World Medical Association. Declaration of Helsinki, Ethical Principles for Medical Research Involving Human Subjects (2004), latest version available electronically at http://www.wma.net/e/policy/b3.htm.

6 Radetzki M, Radetzki M, Juth N: Genes and insurance. Cambridge: Cambridge University Press, 2003.

7 Eriksson S: Should results from genetic research be returned to research subjects and their biological relatives? Trames J Humanities Soc Sci 2004; 8: 46-62.

8 Appelbaum PS, Roth LH, Lidz CW, Benson P, Winslade W: False hopes and best data: consent to research and the therapeutic misconception. Hast Cent Rep 1987; 17: 20-24.

9 Clayton EW: Panel comment: why the use of anonymous samples for research matters. J Law Med Ethics 1995; 23: 375-377.

10 Hoeyer K, Olofsson B-O, Mjörndal T, Lynöe N: Informed consent and biobanks: a population-based study of attitudes towards tissue donation for genetic research. Scand J Public Health 2004; 32: $224-229$.

11 The Nuremberg Code. (1947). BMJ 1996; 313: 1448.

12 Harris J, Woods S: Rights and responsibilities of individuals participating in medical research; in Doyal L, Tobias JS (eds): Informed consent in medical research. London: BMJ Books, 2001, pp 276-282.

13 Committee on Ethical Issues in Medicine of the Royal College of Physicians: Research based on archived information and samples. J Roy Coll Phys London 1999; 33: 264-266.

14 Gustafsson Stolt U, Liss P-E, Svensson T, Ludvigsson J: Attitudes to bioethical issues: A case study of a screening project. Soc Sci Med 2002; 54: 1333-1344.

15 Hoeyer K: 'Science is really needed - that's all I know': informed consent and the non-verbal practices of collecting blood for genetic research in northern Sweden. New Genet Soc 2003; 22: $229-244$. 